\title{
Purtscher's-like retinopathy in acute alcoholic pancreatitis
}

\author{
Praveen Subudhi, ${ }^{1,2}$ Sanghamitra Kanungo, ${ }^{3,4}$ Nageswar Rao Subudhi ${ }^{5}$
}

${ }^{1}$ Department of

Ophthalmology, Ruby Eye

Hospital, Berhampur, Odisha, India

${ }^{2}$ Department of

Ophthalmology, KIMS,

Bhubaneswar, Odisha, India

${ }^{3}$ Kar vision eye hospital,

Bhubaneswar, Odisha, India

${ }^{4}$ Ruby Eye Hospital,

Berhampur, Odisha, India

${ }^{5}$ Department of

Ophthalmology, M.K.C.G

Medical College, Berhampur,

Odisha, India

\section{Correspondence to}

Dr Praveen Subudhi,

subudhipraveen@gmail.com

Accepted 28 August 2016

\section{SUMMARY}

A 28-year-old man presented with a sudden onset of visual loss in both eyes (OU). He had a known history of acute pancreatitis and hepatitis following alcohol abuse. Examination of the anterior segment of the eye revealed non-sustained pupillary light reaction. The fundus showed typical Purtscher's flecken over the posterior pole with multiple cotton wool spots and retinal superficial haemorrhages in OU. Fundus fluorescein angiogram revealed abnormal hypofluorescence in both the posterior poles. Optical coherence tomography (OCT) for Purtscher's flecken showed abnormal retinal thickening with hyper-reflective areas in the inner neurosensory layers. The patient responded favourably to high-dose corticosteroid therapy $(1.5 \mathrm{mg} / \mathrm{kilogram}$ per body weight) with a tapering dose. There was a mild reduction of the ischaemic areas with a corresponding improvement in visual acuity. This case has been presented owing to its rarity and under-reporting. Treatment with corticosteroids yielded favourable results.

\section{BACKGROUND}

Purtscher's-like retinopathy has been attributed to multiple factors including acute pancreatitis. Acute pancreatitis following alcohol abuse is considered
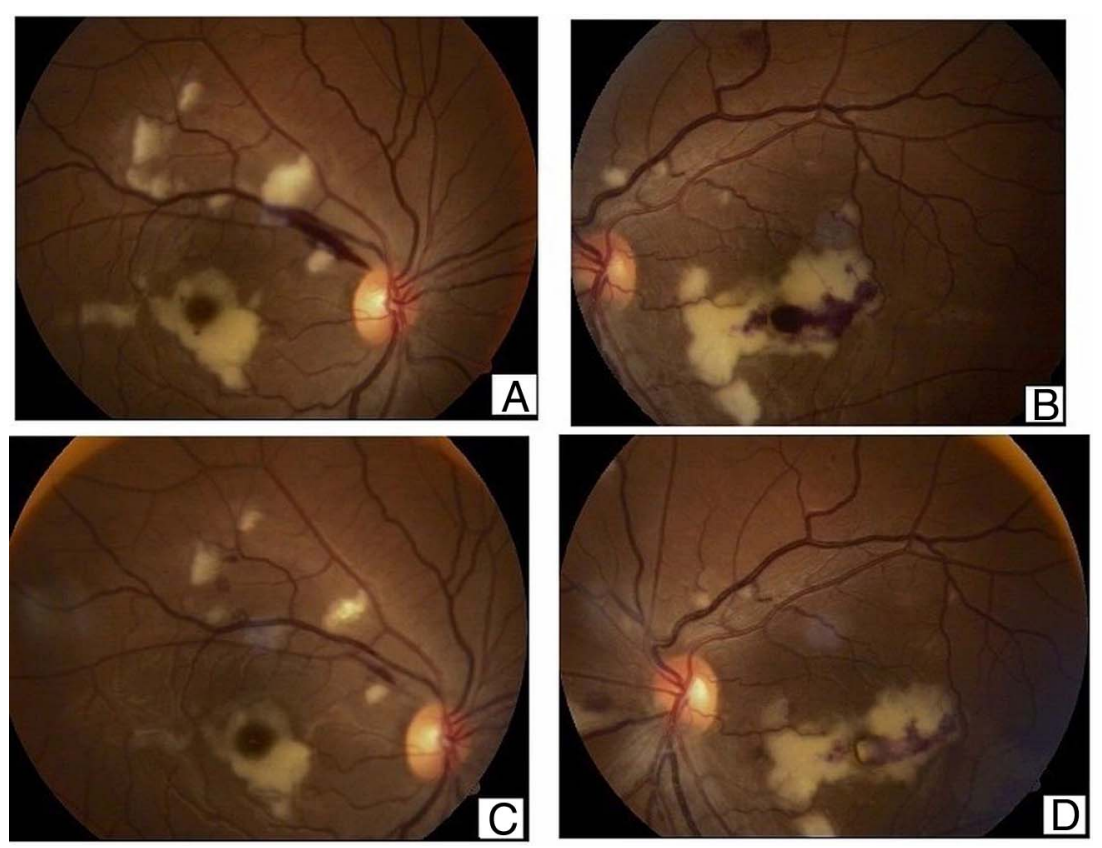

To cite: Subudhi $P$, Kanungo S, Rao Subudhi N. BMJ Case Rep Published online: [please include Day Month Year] doi:10.1136/ bcr-2016-215485

to be very common but visual loss following pancreatic abnormality is rarely observed. However, we presume that the prevalence and incidence of this disease is more common than mentioned in the literature due to under-reporting.

\section{CASE PRESENTATION}

A 28-year-old man presented to our hospital with symptoms of sudden onset bilateral loss of vision. He was a known case of acute pancreatitis and hepatitis following alcohol abuse and was under medical management at a private clinic. His laboratory parameters showed elevated serum trypsin $(850 \mu \mathrm{g} / \mathrm{L})$, lipase (640 units/L), amylase (728 units/L) and alkaline phosphatase (443 units/L) levels. On ocular examination, his best corrected visual acuity was counting fingers at 2 feet using both eyes (OU). An anterior segment examination showed nonsustained pupillary reactions in OU. Posterior segment evaluation revealed clear optical media with whitish lesions over the posterior poles of both eyes (Purtscher's flecken and along the superior vascular arcade, suggestive of cotton wool spots. Superficial nerve fibre layer haemorrhages along the superior vascular arcade in OD and over the posterior pole in OS were also detected

Figure 1 (A and B) Posterior segment evaluation revealed clear optical media with typical Purtscher's flecken over posterior pole of one disc diameter (DD) in the right eye (OD) and two DD in the left eye (OS) associated with multiple cotton wool spots along the superior vascular arcades in both eyes (OU). Superficial nerve fibre layer haemorrhages along the superior vascular arcade in OD and over posterior pole in OS were also detected (C and D); showing reduction of ischaemic zones consequent to steroid therapy. 
Box 1

1. Anassociated contributing illness, such as acute pancreatitis, long bone fracture, orthopaedic surgery, chest compression or crush injury.

2. Multiple areas of polygonal retinal whitening between the retinal arterioles and venules (Purtscher's flecken) and/or superficial cotton wool spots in one or both eyes.

A. Typically restricted to the posterior pole.

B. Accompanied by minimal, if any, retinal haemorrhage.

C. No visible emboli in the large retinal vessels.

D. No direct ocular trauma.
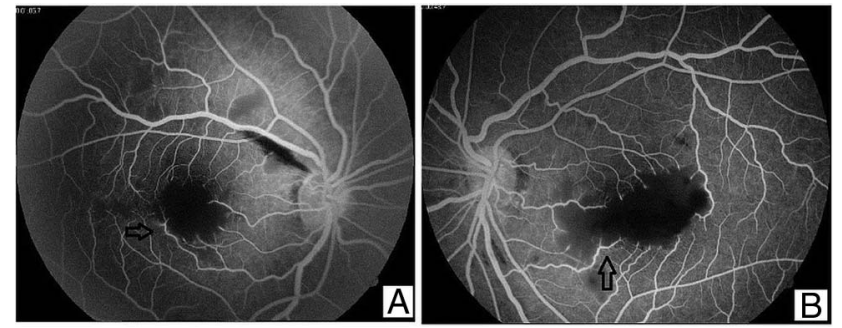

Figure 2 (A) and (B) Fundus fluorescein angiogram showing large ischaemic areas over macula and margins of enlarged hypofluorescent macula had pruning of small vessels suggestive of necrotic inflammation (shown with arrow mark).

(figure 1A,B). As per the diagnostic criteria established by Agrawal and McKibbin ${ }^{1}$ (box 1) Purtscher's-like retinopathy was diagnosed in this case. In terms of the zones of involvement, only zone 1 was involved. The fundus fluorescein angiogram (figure 2A,B) showed symmetrically enlarged foveal avascular zones in $\mathrm{OU}$ corresponding to the central retinal whitening in the coloured fundus photograph. In addition, this test also showed areas of hypofluorescence due to poor capillary filling, corresponding to the cotton wool spots along the vascular arcades. The macular areas showed pruning of vessels. The margins of the central ischaemic zones showed mild dilation of small vessels, with leakage suggestive of necrotic inflammation (figure 2A,B arrow mark). In addition, there was blocked hypofluorescence in areas corresponding to intraretinal haemorrhages in OU. An OCT evaluation (figure 3A,B) (Stratus OCT Carl Zeiss Meditec, Dublin, California, USA) showed abnormal retinal thickening with increased hyper-reflectivity in the inner neurosensory layers of the retina. The foveal contours showed an abnormal slope (OS $>\mathrm{OD})$; however, the central foveal depression was well maintained. The outer hyper-reflective layer was within the normal limits. The patient was started on highdose corticosteroid therapy $(1.5 \mathrm{mg} / \mathrm{kg}$ body weight) with a tapering dose. A favourable response was observed within 4 weeks, with a reduction in the whitish lesions accompanying an improvement in vision (OD 20/80 and OS 20/120; figure 1C,D). Visual acuity was maintained for 2 months, after which the patient was lost to follow-up.

\section{DISCUSSION}

Purtscher's retinopathy was originally associated with severe head trauma. ${ }^{2}$ However, it has also been described in context with other disorders, such as acute pancreatitis, connective tissue disorders, long bone fractures, fat embolism and renal failure. ${ }^{3}$ Cases not associated with trauma but with a similar clinical presentation are termed as Purtscher's-like retinopathies. ${ }^{4}$ The incidence of Purtscher's retinopathy has been reported to be very low ( 0.24 per million population), but similar cases associated with acute pancreatitis are more common than cases associated with trauma. ${ }^{5}$

This paper reports a case of Purtscher's-like retinopathy associated with acute pancreatitis following alcohol abuse. The incidence of sudden visual loss associated with significant retinal changes in cases of acute pancreatitis was found to be $2 \%$. $^{6}$

The activation of the coagulation cascade (complement activation) caused by the release of proteases from the inflamed pancreas results in an accumulation of leucocytes leading to a blockade of intermediate-sized retinal vessels and is thought to be the primary pathophysiology of the condition. ${ }^{7}$ However, the precise pathogenesis of the disease could not be established. Although clinical diagnosis is very important, fundus fluorescein angiography and optical coherence tomography (OCT) are useful adjuncts in the diagnosis of this condition. The pathognomonic Purtscher's flecken is recognised in only 50\% of the total cases reported and was observed in our case. ${ }^{1}$ The retinal nonperfusion surrounding the posterior pole in the fundus fluorescein angiogram corresponding to Purtscher's flecken helps in understanding the disease better and establishes the proposed
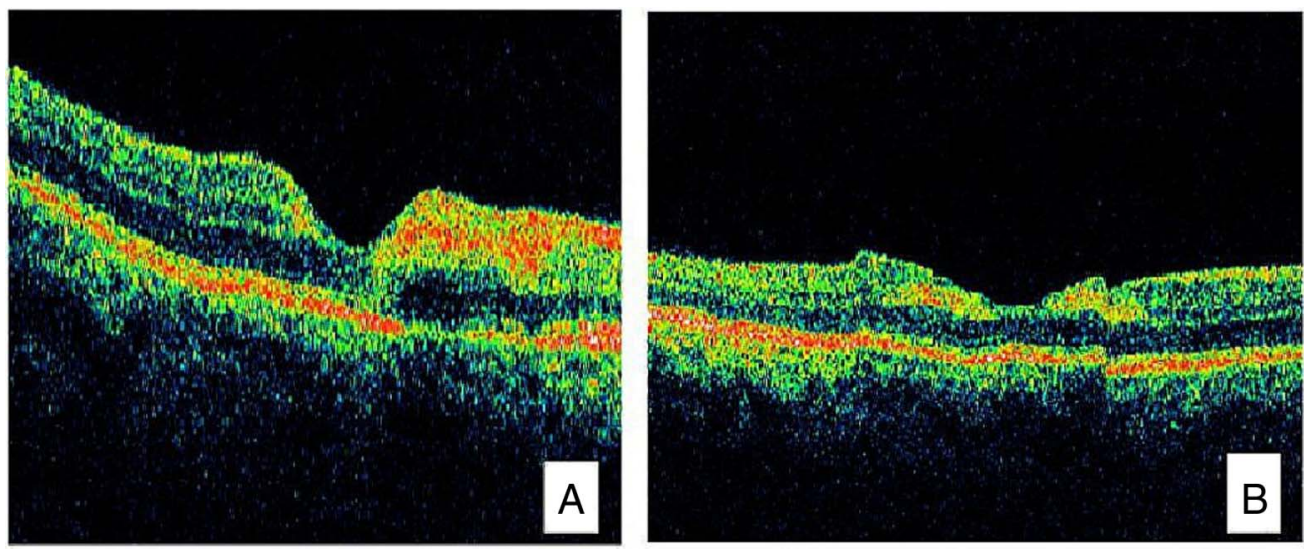

Figure 3 (A and B) OD and OS. Optical coherence tomography showing abnormal hyper-reflective area along inner neurosensory layers associated with irregular foveal surface; however, the foveal contours were well maintained, and the outer hyper-reflective layer was normal. 
hypothesis of ischaemic vasculopathy ${ }^{8}$ in the pathogenesis of the disease. In this case, OCT showed thickened retinal inner layers with hyper-reflectivity that correlated well with the poor retinal perfusion of the inner neurosensory cell layers. ${ }^{9}$ Although the therapeutic role of corticosteroids in improving the visual outcome has been established in previous studies, ${ }^{10} 11$ there was no consensus regarding the appropriate steroid management protocol owing to the few global reports. Therefore, we presume that Purtscher's-like retinopathy is very much under-reported, and, to the best of our knowledge, this is the first case from this region.

\section{Learning points}

- Optical coherence tomography can be used as a non-invasive tool to corroborate clinical diagnosis.

- Ocular examination needs to be performed in all cases of acute pancreatitis to reduce the underdiagnosis of Purtscher's retinopathy.

- Corticosteroids are useful adjuvants for the reduction of necrotic inflammation; however, their therapeutic use is controversial.
Competing interests None declared.

Patient consent Obtained.

Provenance and peer review Not commissioned; externally peer reviewed.

\section{REFERENCES}

1 Agrawal A, McKibbin M. Purtscher's retinopathy: epidemiology, clinical features and outcome. Br J Ophthalmol 2007;91:1456-9.

2 Purtscher 0. Noch unbekannte befunde nach schadeltrauma. Ber Dtsch Ophthalmol Ges 1910;36:294-301.

3 Miguel Al, Henriques F, Azevedo LF, et al. Systematic review of Purtscher's and Purtscher-like retinopathies. Eye (London) 2013;27:1-13.

4 Wu C, Dai R, Dong F, et al. Purtscher-like retinopathy in systemic lupus erythematosus. Am J Ophthalmology 2014;158:1335-1341.e1.

5 Holló G. Frequency of Purtscher's retinopathy. Br J Ophthalmol 2008:92:1159.

6 López-Tizón E, Reinoso-Montalvo C, Mencia-Gutierrez E, et al. [Acute pancreatitis presenting as sudden blindness]. Arch Soc Esp Oftalmol 2006;81:161-3.

7 Mayer C, Khoramnia R. Purtscher-like retinopathy caused by acute pancreatitis. Lancet 2011;378:1653.

8 Papadaki $M$, Lefebvre $P$, Janssens $S$, et al. Bilateral retinal ischemic vasculopathy in a pregnant patient. Retin Cases Brief Rep 2015;9:185-9.

9 Coady PA, Cunningham ET Jr, Vora RA, et al. Spectral domain optical coherence tomography findings in eyes with acute ischaemic retinal whitening. $\mathrm{Br}$ J Ophthalmology 2015:99:586-92.

10 Wang AG, Yen MY, Liu JH. Pathogenesis and neuroprotective treatment in Purtscher's retinopathy. Jpn J Ophthalmology 1998;42:318-22.

11 Atabay C, Kansu T, Nurlu G. Late visual recovery after intravenous methyl prednisolone treatment of Purtscher's retinopathy. Ann Ophthalmology 1993;25:330-3.

Copyright 2016 BMJ Publishing Group. All rights reserved. For permission to reuse any of this content visit http://group.bmj.com/group/rights-licensing/permissions.

BMJ Case Report Fellows may re-use this article for personal use and teaching without any further permission.

Become a Fellow of BMJ Case Reports today and you can:

- Submit as many cases as you like

- Enjoy fast sympathetic peer review and rapid publication of accepted articles

- Access all the published articles

- Re-use any of the published material for personal use and teaching without further permission

For information on Institutional Fellowships contact consortiasales@bmjgroup.com

Visit casereports.bmj.com for more articles like this and to become a Fellow 